\title{
A New Class of Highly Efficient, Non-peptidic Oligoguanidinium Vectors that Selectively Internalize into Mitochondria
}

Jimena Fernández-Carneado, Michiel Van Gool, Vera Martos, Susanna Castel, Pilar Prados, Javier de Mendoza, Ernest Giralt

\section{Supporting Information}

General. Fmoc-protected amino acids were purchased from Advanced Chem Tech. Coupling reagents: 7-azabenzotriazol-1-yloxytris(pyrrolidino)phosphonium hexafluorophosphate (PyAOP) was purchased from Applied Biosystems; Rink amide MBHA resin was purchased from NovaBiochem; 1-hydroxy-7-azabenzotriazole (HOAt) was purchased from GL Biochem; 2-(1Hbenzotriazol-1-yl)-1,1,3,3-tetramethyluronium tetrafluoroborate (TBTU) and 1hydroxybenzotriazole (HOBT) were purchased from Albatros Chem Inc. Solvents: trifluoroacetic acid (TFA), piperidine, dimethylformamide (DMF), dichloromethane, acetonitrile, methanol and diethyl ether were purchased from SDS. Reagents: potassium thioacetate, ammonium hexafluorophosphate, methanesulfonic anhydride, tributylphosphine, cesium carbonate and cystamine were purchased from Aldrich; methanesulfonic acid, $N$-methyl morpholine (NMM) and 5(6)-carboxyfluorescein (CF) were purchased from Acros; benzotriazol-1-yloxytripyrrolidinophosphonium hexafluorophosphate (PyBOP) was purchased from NovaBiochem; diisopropylcarbodiimide (DIC) and N,N-diisopropylethylamine (DIEA) were purchased from Merck; triisopropylsilane (TIS) and potassium hexafluorophosphate were purchased from Fluka.

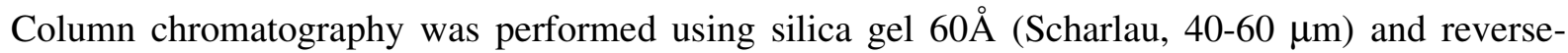
phase silica gel LiChroprep ${ }^{\circledR}$ RP-18 (Merck, 25-40 $\mu \mathrm{m}$ ). Thin layer chromatography (TLC) was performed on Alugram Sil G/UV254-coated aluminium sheets (Macherey-Nagel) with detection by $\mathrm{UV}$ at 254/365 $\mathrm{nm}$ and/or with bromocresol green (1.4 $\mathrm{mM}$ in EtOH, 4\% 0.1 M NaOH). Melting points were determined on a Gallenkamp apparatus. Optical rotations $[\alpha]^{20}{ }_{D}$ were determined on a Perkin-Elmer $241 \mathrm{MC}$ polarimeter, using a quartz cell $(1 \mathrm{dm})$ at $298 \mathrm{~K}\left(\mathrm{Na}_{\mathrm{D}} 589 \mathrm{~nm}\right) .{ }^{1} \mathrm{H}$ and ${ }^{13} \mathrm{C}$ NMR spectra were recorded on Bruker AMX-300 and DRX-500 spectrometers at $298 \mathrm{~K}$. Chemical

shifts $(\delta)$ are expressed in ppm relative to the solvent residual peak. ${ }^{13} \mathrm{C}$ NMR spectra were assigned using DEPT (distortionless enhancement by polarization transfer) experiments. Mass spectra by fast atom bombardment $(\mathrm{FAB})$ were recorded on a VG AutoSpec spectrometer using $m$-nitrobenzyl alcohol as matrix and by matrix-assisted laser desorption ionization-time-of-flight (MALDI-TOF) method on a REFLEX spectrometer using ditranol as matrix. Elemental analyses were performed on a LECO CHNS 932 microanalyser. 
Synthesis.

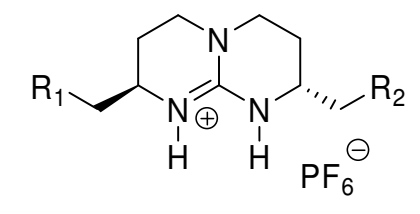

$7 \quad R_{1}=$ OTBDPS; $R_{2}=$ OMs

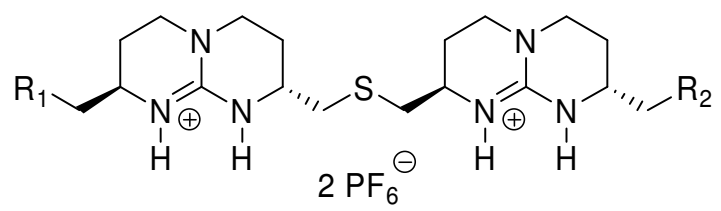

$8 \mathrm{R}_{1}=$ OTBDPS; $\mathrm{R}_{2}=\mathrm{OH}$

$10 \mathrm{R}_{1}=$ OTBDPS; $\mathrm{R}_{2}=\mathrm{OMs}$

Compound 8. A solution of mesylate $7^{1}(1.90 \mathrm{~g}, 2.87 \mathrm{mmol})$ and potassium thioacetate $(0.426 \mathrm{~g}$, $3.73 \mathrm{mmol})$ in a mixture of THF $(40 \mathrm{ml})$ and water $(15 \mathrm{ml})$ was refluxed for $24 \mathrm{~h}$. After cooling to room temperature $\mathrm{MsOH}(0.930 \mathrm{ml}, 14.35 \mathrm{mmol})$ was added and the mixture was refluxed again for $24 \mathrm{~h}$. The reaction mixture was cooled to room temperature, water $(150 \mathrm{ml})$ and $\mathrm{Et}_{2} \mathrm{O}(150 \mathrm{ml})$ were added and after extraction both phases were separated. The organic phase was extracted once again with water $(30 \mathrm{ml})$. The combined aqueous phases were washed with $\mathrm{CHCl}_{3}(150 \mathrm{ml})$ and $\mathrm{Et}_{2} \mathrm{O}$ $(150 \mathrm{ml})$. After concentration of about $30 \%$ of the aqueous layer, $\mathrm{KHCO}_{3}(1.72 \mathrm{~g}, 17.22 \mathrm{mmol})$ was added and the solvent evaporated to dryness. Then $\mathrm{MeOH}(100 \mathrm{ml})$ was added, the precipitate was removed by filtration and the solvent evaporated. This procedure was repeated a few times with increasing amounts of $\mathrm{CH}_{2} \mathrm{Cl}_{2}$, until pure $\mathrm{CH}_{2} \mathrm{Cl}_{2}(20 \mathrm{ml})$, resulting in a slightly yellow solid (0.93 g). To a solution of this product and $\mathrm{Cs}_{2} \mathrm{CO}_{3}(840 \mathrm{mg}, 2.58 \mathrm{mmol})$ in $\mathrm{MeOH}(30 \mathrm{ml})$ tributylphosphine $(415 \mu \mathrm{l}, 1.55 \mathrm{mmol})$ was added and the mixture was stirred for $40 \mathrm{~min}$ at room temperature. Then a solution of mesylate $7(1.71 \mathrm{~g}, 2.58 \mathrm{mmol})$ in THF $(50 \mathrm{ml})$ was added and the mixture was stirred for $1 \mathrm{~h}$ at the same temperature. After evaporation of the solvent, $\mathrm{CH}_{2} \mathrm{Cl}_{2}$ and $0.1 \mathrm{M}$ aq $\mathrm{NH}_{4} \mathrm{PF}_{6}$ were added. After phase separation the aqueous layer was extracted once more with $\mathrm{CH}_{2} \mathrm{Cl}_{2}$ and the combined organic phases were filtered over cotton and concentrated to dryness. The crude residue was purified by column chromatography on silica gel $\left(\mathrm{CH}_{2} \mathrm{Cl}_{2} / \mathrm{MeOH}\right.$, $4 \% \rightarrow 6 \%)$, yielding $8(1.99 \mathrm{~g}, 75 \%)$ as dihexafluorophosphate salt. $\mathrm{mp} 101^{\circ} \mathrm{C} ;[\alpha]_{\mathrm{D}}{ }^{20}-96(c=0.4$, $\mathrm{MeOH}) ;{ }^{1} \mathrm{H} \mathrm{NMR}\left(\mathrm{CDCl}_{3}, 300 \mathrm{MHz}\right) \delta$ 7.67-7.60 (m, 4H), 7.45-7.35 (m, 6H), 6.48 (br s, 1H), 6.33 (m, 2H), 6.22 (br s, 1H), 3.73-3.20 (m, 16H), 2.90-2.45 (m, 4H), 2.15-1.30 (m, 8H), 1.08 (s, 9H); ${ }^{13} \mathrm{C} \mathrm{NMR}\left(\mathrm{CDCl}_{3}, 75 \mathrm{MHz}, \mathrm{DEPT}\right) \delta 150.7(\mathrm{C}), 150.5(\mathrm{C}), 135.4(\mathrm{CH}), 132.7(\mathrm{C}), 132.5(\mathrm{C}), 129.9$ $(\mathrm{CH}), 127.8(\mathrm{CH}), 65.3\left(\mathrm{CH}_{2}\right), 64.4\left(\mathrm{CH}_{2}\right), 50.2(\mathrm{CH}), 49.8(\mathrm{CH}), 47.6(\mathrm{CH}), 47.1(\mathrm{CH}), 45.3$ $\left(\mathrm{CH}_{2}\right), 44.8\left(\mathrm{CH}_{2}\right), 36.0\left(\mathrm{CH}_{2}\right), 35.7\left(\mathrm{CH}_{2}\right), 26.7\left(\mathrm{CH}_{3}\right), 26.5\left(\mathrm{CH}_{2}\right), 25.7\left(\mathrm{CH}_{2}\right), 22.4\left(\mathrm{CH}_{2}\right), 22.2$ $\left(\mathrm{CH}_{2}\right), 19.0(\mathrm{C}) ; \mathrm{HRMS}\left(\mathrm{FAB}^{+}\right) \mathrm{m} / z 635.3574(\mathrm{calcd} 635.3563)\left[\mathrm{M}-\mathrm{HPF}_{6}-\mathrm{PF}_{6}\right]^{+} ; \mathrm{MS}\left(\mathrm{FAB}^{+}\right) \mathrm{m} / z$ $781.2(15)\left[\mathrm{M}-\mathrm{PF}_{6}\right]^{+}, 635.2(100)\left[\mathrm{M}-\mathrm{HPF}_{6}-\mathrm{PF}_{6}\right]^{+}$; elemental analysis calcd (\%) for $\mathrm{C}_{34} \mathrm{H}_{52} \mathrm{~F}_{12} \mathrm{~N}_{6} \mathrm{O}_{2} \mathrm{P}_{2} \mathrm{SSi}$ : C 44.06, H 5.65, N 9.07, S 3.46; found: C 44.85, H 5.33, N 8.85, S 3.89.

Compound 10. To a solution of alcohol $8(0.270 \mathrm{~g}, 0.291 \mathrm{mmol})$ and NMM (96 $\mu 1,0.874 \mathrm{mmol})$ in THF $(8 \mathrm{ml})$ at $0^{\circ} \mathrm{C}$ was added a solution of $\mathrm{Ms}_{2} \mathrm{O}(0.105 \mathrm{~g}, 0.439 \mathrm{mmol})$ in THF $(2 \mathrm{ml})$ and the 
mixture was stirred for $2 \mathrm{~h}$ at this temperature. The solvent was evaporated, $\mathrm{CH}_{2} \mathrm{Cl}_{2}(40 \mathrm{ml})$ and 0.1 $\mathrm{M}$ aq $\mathrm{NH}_{4} \mathrm{PF}_{6}(20 \mathrm{ml})$ were added and, after extraction, the organic phase was separated. The aqueous phase was extracted once more with $\mathrm{CH}_{2} \mathrm{Cl}_{2}(10 \mathrm{ml})$. The combined organic layers were filtered over cotton, concentrated to dryness and the residue was purified by column chromatography on silica gel $\left(\mathrm{CH}_{2} \mathrm{Cl}_{2} / \mathrm{MeOH}, 5 \%\right)$, affording 10 (276 $\mathrm{mg}$, 94\%) as dihexafluorophosphate salt. mp $78^{\circ} \mathrm{C} ;[\alpha]_{\mathrm{D}}{ }^{20}-83\left(c=0.5, \mathrm{CHCl}_{3}\right) ;{ }^{1} \mathrm{H} \mathrm{NMR}\left(\mathrm{CDCl}_{3}, 500 \mathrm{MHz}\right) \delta$ 7.63-7.65 (m, 4H, Ar), 7.39-7.45 (m, 6H, Ar), 6.32 (s, 1H, NH), 6.26 (s, 1H, NH), 6.08 (s, 1H, NH), 4.21-4.24 (AB-system, $J_{\mathrm{AB}}=10.6 \mathrm{~Hz}, 1 \mathrm{H}, \mathrm{CH}_{2} \mathrm{O}$ ), 4.07-4.10 (AB-system, $J_{\mathrm{AB}}=10.6 \mathrm{~Hz}, 1 \mathrm{H}$, $\mathrm{CH}_{2} \mathrm{O}$ ), 3.72-3.75 (m, $\left.1 \mathrm{H}\right), 3.64-3.68\left(\mathrm{AB}\right.$-system, $\left.J_{\mathrm{AB}}=10.4 \mathrm{~Hz}, 1 \mathrm{H}, \mathrm{CH}_{2} \mathrm{O}\right), 3.59-3.61(\mathrm{~m}, 4 \mathrm{H})$, 3.33-3.44 (m, 4H), 3.24-3.40 (m, $1 \mathrm{H}), 3.02\left(\mathrm{~s}, 3 \mathrm{H}, \mathrm{CH}_{3} \mathrm{SO}_{2}\right), 2.82-2.88\left(\mathrm{~m}, 2 \mathrm{H}, \mathrm{CH}_{2} \mathrm{~S}\right), 2.66-2.72$ (m, $\left.2 \mathrm{H}, \mathrm{CH}_{2} \mathrm{~S}\right), 2.05-2.14\left(\mathrm{~m}, 4 \mathrm{H}, \mathrm{CH}_{2} \beta\right), 1.87-2.04\left(\mathrm{~m}, 4 \mathrm{H}, \mathrm{CH}_{2} \beta\right), 1.06\left[\mathrm{~s}, 9 \mathrm{H}, \mathrm{C}\left(\mathrm{CH}_{3}\right)_{3}\right] ;{ }^{13} \mathrm{C}$ NMR $\left(\mathrm{CDCl}_{3}, 125 \mathrm{MHz}, \mathrm{DEPT}\right) \delta 151.3(\mathrm{C}), 151.2(\mathrm{C}), 135.6(2 \times \mathrm{CH}), 132.8(\mathrm{CH}), 130.0(\mathrm{CH})$, $127.9(\mathrm{CH}), 69.1\left(\mathrm{CH}_{2}\right), 65.5\left(\mathrm{CH}_{2}\right), 49.7(\mathrm{CH}), 47.4(\mathrm{CH}), 47.2(\mathrm{CH}), 46.4\left(\mathrm{CH}_{2}\right), 45.4\left(\mathrm{CH}_{2}\right), 45.1$ $\left(\mathrm{CH}_{2}\right), 45.0\left(\mathrm{CH}_{2}\right), 44.8\left(\mathrm{CH}_{2}\right), 37.3\left(\mathrm{CH}_{2}\right), 37.0\left(\mathrm{CH}_{3}\right), 36.9\left(\mathrm{CH}_{2}\right), 26.8\left(\mathrm{CH}_{2}\right), 26.1\left(\mathrm{CH}_{2}\right), 22.6$ $\left(\mathrm{CH}_{3}\right), 22.2\left(\mathrm{CH}_{3}\right), 19.2(\mathrm{C}) ; \mathrm{MS}\left(\mathrm{FAB}^{+}\right) \mathrm{m} / z 713.5\left[\mathrm{M}-\mathrm{HPF}_{6}-\mathrm{PF}_{6}\right]^{+}, 859.3\left[\mathrm{M}-\mathrm{PF}_{6}\right]^{+}$; elemental analysis calcd (\%) for $\mathrm{C}_{35} \mathrm{H}_{54} \mathrm{~F}_{12} \mathrm{~N}_{6} \mathrm{O}_{4} \mathrm{P}_{2} \mathrm{~S}_{2}$ : C 41.83, H 5.42, N 8.36, S 6.38; found: C 41.92, H 5.33, N 8.22, S 6.34 .

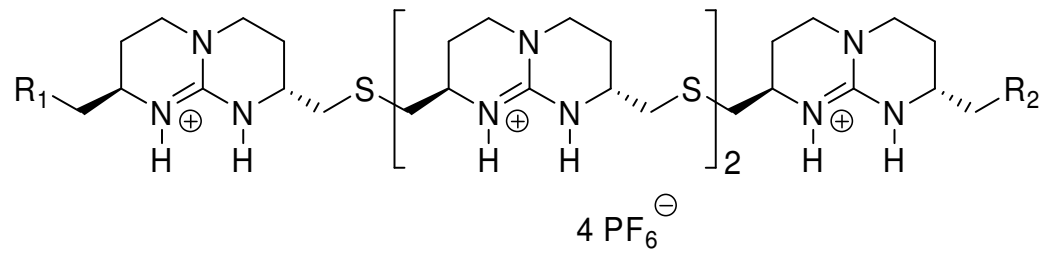

$$
\begin{array}{ll}
11 & \mathrm{R}_{1}=\text { OTBDPS } ; \mathrm{R}_{2}=\mathrm{OH} \\
12 & \mathrm{R}_{1}=\text { OTBDPS; } \mathrm{R}_{2}=\mathrm{OMS}
\end{array}
$$

Compound 11. A solution of mesylate $10(640 \mathrm{mg}, 0.637 \mathrm{mmol})$ and potassium thioacetate (370 $\mathrm{mg}, 3.18 \mathrm{mmol})$ in a mixture of THF $(25 \mathrm{ml})$ and water $(10 \mathrm{ml})$ was refluxed for $18 \mathrm{~h}$. After cooling to room temperature $\mathrm{MsOH}(0.62 \mathrm{ml}, 9.55 \mathrm{mmol})$ was added and the mixture was refluxed for $24 \mathrm{~h}$. The reaction was cooled to room temperature, water $(150 \mathrm{ml})$ and $\mathrm{Et}_{2} \mathrm{O}(150 \mathrm{ml})$ were added and, after extraction, the phases were separated. The aqueous phase was washed with $\mathrm{CHCl}_{3}(150 \mathrm{ml})$ and once again with $\mathrm{Et}_{2} \mathrm{O}(150 \mathrm{ml})$. After evaporation of about $50 \%$ of the aqueous layer, $\mathrm{KHCO}_{3}$ $(1.1 \mathrm{~g}, 10.8 \mathrm{mmol})$ was added and the solvent was evaporated to dryness. Then $\mathrm{MeOH}(100 \mathrm{ml})$ and $\mathrm{CH}_{2} \mathrm{Cl}_{2}(20 \mathrm{ml})$ were added, the precipitate was removed by filtration and the solvent was evaporated. This procedure was repeated a few times with increasing amounts of $\mathrm{CH}_{2} \mathrm{Cl}_{2}$, until pure $\mathrm{CH}_{2} \mathrm{Cl}_{2}$ (150 ml), resulting in a slightly yellow solid $(352 \mathrm{mg})$. To a solution of this product and $\mathrm{Cs}_{2} \mathrm{CO}_{3}(170 \mathrm{mg}, 0.50 \mathrm{mmol})$ in $\mathrm{MeOH}(12 \mathrm{ml})$ tributylphosphine $(80 \mu \mathrm{l}, 0.30 \mathrm{mmol})$ was added 
and the reaction was stirred for $30 \mathrm{~min}$ at room temperature. Then a solution of mesylate $\mathbf{1 0}$ (503 $\mathrm{mg}, 0.50 \mathrm{mmol})$ in THF (12 ml) was added and the mixture was stirred for $1 \mathrm{~h}$. After evaporation of the solvent, $\mathrm{CH}_{2} \mathrm{Cl}_{2}(70 \mathrm{ml})$ and $0.1 \mathrm{M}$ aq $\mathrm{NH}_{4} \mathrm{PF}_{6}(50 \mathrm{ml})$ were added. After phases separation, the aqueous layer was extracted with $\mathrm{CH}_{2} \mathrm{Cl}_{2}(2 \times 20 \mathrm{ml})$ and the combined organic phases were filtered over cotton and concentrated to dryness. The crude residue was purified by column chromatography on silica gel $\left(\mathrm{CH}_{2} \mathrm{Cl}_{2} / \mathrm{MeOH}, 3 \% \rightarrow 5 \%\right)$, yielding $11(625 \mathrm{mg}, 61 \%)$ as tetrahexafluorophosphate salt. mp $88^{\circ} \mathrm{C} ;[\alpha]_{\mathrm{D}}{ }^{20}-132(c=0.5, \mathrm{MeOH}) ;{ }^{1} \mathrm{H}$ NMR (acetone- $\left.d_{6}, 500 \mathrm{MHz}\right) \delta 7.73-7.70(\mathrm{~m}, 4 \mathrm{H})$, 7.53-7.45 (m, 6H), 7.32 (m, 4H), 7.29 (br s, 1H), 7.26 (br s, 1H), 7.15 (br s, 1H), 7.11 (br s, 1H), 3.85-3.65 (m, 10H), 3.62-3.48 (m, 18H), 3.07-2.98 (m, 6H), 2.78-2.68 (m, 6H), 2.28-1.80 (m, 16H), $1.08(\mathrm{~s}, 9 \mathrm{H}) ;{ }^{13} \mathrm{C}$ NMR (acetone- $\left.d_{6}, 125 \mathrm{MHz}, \mathrm{DEPT}\right) \delta 151.3(2 \times \mathrm{C}), 151.2(\mathrm{C}), 135.7(2 \times \mathrm{CH})$, $133.2(\mathrm{C}), 133.1(\mathrm{C}), 130.3(2 \times \mathrm{CH}), 128.2(2 \times \mathrm{CH}), 66.3\left(\mathrm{CH}_{2}\right), 64.4\left(\mathrm{CH}_{2}\right), 51.1(\mathrm{CH}), 50.6$ $(\mathrm{CH}), 48.2(\mathrm{CH}), 48.1(\mathrm{CH}), 48.0(\mathrm{CH}), 45.7\left(\mathrm{CH}_{2}\right), 45.5\left(\mathrm{CH}_{2}\right), 45.4\left(\mathrm{CH}_{2}\right), 45.3\left(\mathrm{CH}_{2}\right), 36.3$ $\left(\mathrm{CH}_{2}\right), 36.2\left(\mathrm{CH}_{2}\right), 36.1\left(\mathrm{CH}_{2}\right), 26.6\left(\mathrm{CH}_{3}\right), 26.0\left(\mathrm{CH}_{2}\right), 25.8\left(\mathrm{CH}_{2}\right), 25.7\left(\mathrm{CH}_{2}\right), 22.8\left(\mathrm{CH}_{2}\right), 22.7$ $\left(\mathrm{CH}_{2}\right), 19.15(\mathrm{C}) ; \mathrm{HRMS}\left(\mathrm{FAB}^{+}\right) \mathrm{m} / z 1029.5512($ calcd 1029.5537$)\left[\mathrm{M}-\mathrm{HPF}_{6}-\mathrm{PF}_{6}\right]^{+} ; \mathrm{MS}\left(\mathrm{FAB}^{+}\right)$ $m / z 1467.5(92)\left[\mathrm{M}-\mathrm{PF}_{6}\right]^{+}, 1321.7(100)\left[\mathrm{M}-\mathrm{HPF}_{6}-\mathrm{PF}_{6}\right]^{+}, 1175.5(78)\left[\mathrm{M}-2 \mathrm{HPF}_{6}-\mathrm{PF}_{6}\right]^{+}$, 1029.5 (76) $\left[\mathrm{M}-3 \mathrm{HPF}_{6}-\mathrm{PF}_{6}\right]^{+}$; elemental analysis calcd $(\%)$ for $\mathrm{C}_{52} \mathrm{H}_{84} \mathrm{~F}_{24} \mathrm{~N}_{12} \mathrm{O}_{2} \mathrm{P}_{4} \mathrm{~S}_{3} \mathrm{Si}$ : C 38.71, H 5.19, N 10.42, S 5.96; found: C 39.14, H 4.89, N 10.28, S 6.50.

Compound 12. To a solution of alcohol 11 (107 mg, $0.066 \mathrm{mmol})$ and NMM (58 $\mu 1,0.52 \mathrm{mmol})$ in $\mathrm{CH}_{3} \mathrm{CN}(2 \mathrm{ml})$ was added a solution of $\mathrm{Ms}_{2} \mathrm{O}(105 \mathrm{mg}, 0.20 \mathrm{mmol})$ in $\mathrm{CH}_{3} \mathrm{CN}(1 \mathrm{ml})$ and the mixture was stirred for $2 \mathrm{~h}$ at room temperature. The solvent was evaporated, $\mathrm{CH}_{2} \mathrm{Cl}_{2}$ and $0.1 \mathrm{M}$ aq $\mathrm{NH}_{4} \mathrm{PF}_{6}$ were added and, after extraction, the organic phase was separated. The aqueous phase was extracted once more with $\mathrm{CH}_{2} \mathrm{Cl}_{2}$. The combined organic layers were filtered over cotton and concentrated to dryness and the residue was purified by column chromatography on silica gel $\left(\mathrm{CH}_{2} \mathrm{Cl}_{2} / \mathrm{MeOH}, 3 \% \rightarrow 5 \%\right)$, affording $12(107 \mathrm{mg}, 95 \%)$ as tetrahexafluorophosphate salt. mp $111^{\circ} \mathrm{C} ;[\alpha]_{\mathrm{D}}{ }^{20}-117(c=0.4, \mathrm{MeOH}) ;{ }^{1} \mathrm{H}$ NMR (acetone- $\left.d_{6}, 500 \mathrm{MHz}\right) \delta 7.73-7.70(\mathrm{~m}, 4 \mathrm{H}), 7.53-$ 7.45 (m, 6H), 7.40 (br s, 1H), 7.20 (m, 6H), 7.02 (br s, 1H), 4.47 (dd, $J=4.1,10.5 \mathrm{~Hz}, 1 \mathrm{H}), 4.29$ $(\mathrm{dd}, J=7.5,10.5 \mathrm{~Hz}, 1 \mathrm{H}), 4.00(\mathrm{~m}, 1 \mathrm{H}), 3.85-3.70(\mathrm{~m}, 9 \mathrm{H}), 3.61-3.48(\mathrm{~m}, 16 \mathrm{H}), 3.20(\mathrm{~s}, 3 \mathrm{H}), 3.07-$ $2.98(\mathrm{~m}, 6 \mathrm{H}), 2.78-2.69(\mathrm{~m}, 6 \mathrm{H}), 2.30-2.18(\mathrm{~m}, 8 \mathrm{H}), 2.04-1.90(\mathrm{~m}, 8 \mathrm{H}), 1.08(\mathrm{~s}, 9 \mathrm{H}) ;{ }^{13} \mathrm{C} \mathrm{NMR}$ (acetone- $\left.d_{6}, 125 \mathrm{MHz}, \mathrm{DEPT}\right) \delta 151.9(\mathrm{C}), 151.8(2 \times \mathrm{C}), 136.3(\mathrm{CH}), 136.2(\mathrm{CH}), 133.7(\mathrm{C}), 133.6$ (C), $130.9(2 \times \mathrm{CH}), 128.8(2 \times \mathrm{CH}), 71.7\left(\mathrm{CH}_{2}\right), 66.9\left(\mathrm{CH}_{2}\right), 51.2(\mathrm{CH}), 48.7(\mathrm{CH}), 48.6(\mathrm{CH})$, $46.0\left(\mathrm{CH}_{2}\right), 45.9\left(\mathrm{CH}_{2}\right), 45.5\left(\mathrm{CH}_{2}\right), 37.2(\mathrm{CH}), 36.6\left(\mathrm{CH}_{2}\right), 27.2\left(\mathrm{CH}_{3}\right), 26.3\left(\mathrm{CH}_{2}\right), 26.2\left(\mathrm{CH}_{2}\right)$, $23.2\left(\mathrm{CH}_{2}\right), 22.6\left(\mathrm{CH}_{2}\right), 19.7(\mathrm{C}) ; \mathrm{HRMS}\left(\mathrm{FAB}^{+}\right) \mathrm{m} / z 1545.4408$ (calcd 1545.4472) [M - $\left.\mathrm{PF}_{6}\right]^{+} ; \mathrm{MS}$ $\left(\mathrm{FAB}^{+}\right) m / z 1545.8(100)\left[\mathrm{M}-\mathrm{PF}_{6}\right]^{+}, 1399.6(70)\left[\mathrm{M}-\mathrm{HPF}_{6}-\mathrm{PF}_{6}\right]^{+}, 1253.7$ (30) $\left[\mathrm{M}-2 \mathrm{HPF}_{6}-\right.$ $\left.\mathrm{PF}_{6}\right]^{+}, 1107.5(6)\left[\mathrm{M}-3 \mathrm{HPF}_{6}-\mathrm{PF}_{6}\right]^{+}$. 


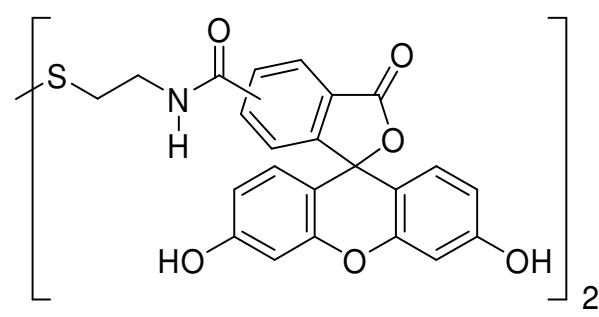

13

Compound 13. A mixture of 5(6)-carboxyfluorescein (575 mg, $1.528 \mathrm{mmol})$, cystamine $(0.172 \mathrm{~g}$, $0.76 \mathrm{mmol}), \operatorname{pyBOP}(0.875 \mathrm{~g}, 1.68 \mathrm{mmol})$, DIEA $(1.2 \mathrm{ml}, 6.88 \mathrm{mmol})$ and HOBT (catalytic amount) in $\mathrm{DMF}(30 \mathrm{ml})$ was stirred overnight at room temperature. The solvent was evaporated, $\mathrm{Et}_{2} \mathrm{O}$ was added and, after sonication, the organic layer was removed. Then $\mathrm{CHCl}_{3}$ was added to precipitate the product, which was filtered off and purified by column chromatography on silica gel $\left(\mathrm{CH}_{2} \mathrm{Cl}_{2} / \mathrm{MeOH} / \mathrm{AcOH}, 92: 8: 2\right)$, resulting in $\mathbf{1 3}$ (330 mg, 50\%).

General Procedure for the Syntheses of CF-RKKRRQRRR-NH2 (CF-Tat) and CFRQIKIWFQNRRMKWKK-NH $\mathbf{H}_{2}$ (CF-Antp). The syntheses of $\mathrm{H}_{2} \mathrm{~N}$-RKKRRQRRR-Rink Amide MBHA resin and $\mathrm{H}_{2} \mathrm{~N}$-RQIKIWFQNRRMKWKK-Rink Amide MBHA resin were carried out automatically on an Applied Biosystems 433A Peptide Synthesizer. Rink Amide MBHA resin (0.1 mmol, $128 \mathrm{mg}$; Initial loading of the resin $=0.78 \mathrm{mmol} / \mathrm{g}$ ), $10 \mathrm{eq}$ of amino acid and a solution of $0.45 \mathrm{M}$ TBTU/HOBT and $2 \mathrm{M}$ DIEA in N-methylpyrrolidine (NMP) were used for coupling reactions. After completion of the sequences, $\mathrm{H}_{2} \mathrm{~N}$ $\mathrm{R}(\mathrm{Pbf}) \mathrm{K}(\mathrm{Boc}) \mathrm{K}(\mathrm{Boc}) \mathrm{R}(\mathrm{Pbf}) \mathrm{R}(\mathrm{Pbf}) \mathrm{Q}(\mathrm{Trt}) \mathrm{R}(\mathrm{Pbf}) \mathrm{R}(\mathrm{Pbf}) \mathrm{R}(\mathrm{Pbf})-\mathrm{Rink}$ Amide $\mathrm{MBHA}$ and $\mathrm{H}_{2} \mathrm{~N}-$ R(Pbf)Q(Trt)IK(Boc)IW(Boc)FQ(Trt)N(Trt)R(Pbf)R(Pbf)MK(Boc)W(Boc)K(Boc)K(Boc)-Rink Amide MBHA, peptide-resins were treated with a solution of CF (5 eq), PyAOP (5 eq), HOAt (5 eq) and DIEA (10 eq) dissolved in $\mathrm{DMF} / \mathrm{CH}_{2} \mathrm{Cl}_{2}$ 9/1, preactivated for $10 \mathrm{~min}$ before addition, and stirred for $1.5 \mathrm{~h}$. The labeled peptides were cleaved from the resin by treatment with $81.5 \%$ TFA, $5 \%$ thioanisole, 5\% water, 5\% EDT, $1 \%$ TIS, 5\% phenol for $4 \mathrm{~h}$. The labeled peptides were identified at $\lambda=443 \mathrm{~nm}$ by analytical RP-HPLC [Waters 996 photodiode array detector equipped with the Waters 2695 separation module, Symmetry column $(\mathrm{C} 18,5 \mu \mathrm{m}, 4.6 \times 150 \mathrm{~mm})$ and Millennium software; Flow $=1 \mathrm{ml} / \mathrm{min}$; Gradient $=5-100 \% \mathrm{~B}$ in $15 \mathrm{~min}(\mathrm{~B}=0.036 \%$ TFA in acetonitrile)]. CF-peptides were purified by semi-preparative RP-HPLC [Waters 2487 Dual $\lambda$ Absorbance Detector equipped with the Waters 2700 Sample Manager, Waters 600 Controller, Waters Fraction Collector, Symmetry® column $(\mathrm{C} 18,5 \mu \mathrm{m}, 30 \times 100 \mathrm{~mm})$ and Millennium chromatography manager software; Flow $=10 \mathrm{ml} / \mathrm{min}$; Gradient $=5-20 \% \mathrm{D}$ in $5 \mathrm{~min} ; 20-70 \% \mathrm{D}$ in $30 \mathrm{~min} ; 70-100 \% \mathrm{D}$ in $5 \mathrm{~min}$ ( $\mathrm{D}=0.1 \%$ TFA in acetonitrile)] and further characterized by MALDITOF MS (Vogayer-DE RP MALDI-TOF, PE Biosystems with a $\mathrm{N}_{2}$ laser of $337 \mathrm{~nm}$ ). 
CF-RKKRRQRRR-NH $:$ m/z 1698 (Calcd 1697) $[\mathrm{M}+\mathrm{H}]^{+}, 1720[\mathrm{M}+\mathrm{Na}]^{+}$.

CF-RQIKIWFQNRRMKWKK-NH $2: m / z, 1303\left(\right.$ Calcd 2604) $[\mathrm{M}+2 \mathrm{H}]^{2+}, 869[\mathrm{M}+3 \mathrm{H}]^{3+}, 652$ $[\mathrm{M}+4 \mathrm{H}]^{4+}, 522[\mathrm{M}+5 \mathrm{H}]^{5+}$.

Combination of experimental absorption values of the different CF-peptides obtained by UV at 490 and $443 \mathrm{~nm}$ and Amino Acid Analysis allowed the exact concentration to be determined for each sample.

MTT assay. For each assay $7 \times 10^{3}$ cells $/ \mathrm{cm}^{2}$ were seeded in a 96-well plate (Nalge Nunc International) and cultured for $24 \mathrm{~h}$. Compounds were added at concentrations ranging from $1 \mu \mathrm{M}$ to $50 \mu \mathrm{M}$. Cells were incubated for $24 \mathrm{~h}$ at $37^{\circ} \mathrm{C}$ under a $5 \% \mathrm{CO}_{2}$ atmosphere. After $22 \mathrm{~h}$, MTT was added at $0.5 \mathrm{mg} / \mathrm{ml}$ final concentration. The cells with peptide and MTT were incubated for a further $2 \mathrm{~h}$ and then the medium was discarded. Isopropanol was added to dissolve the formazan product and $30 \mathrm{~min}$ later absorbance was measured at $\lambda=570 \mathrm{~nm}$. The cell viability is expressed as a percentage ratio of cells treated with the different compounds against untreated cells.

\section{References}

1. Breccia, P., Van Gool, M., Perez-Fernandez, R., Martin-Santamaria, S., Gago, F., Prados, P. \& de Mendoza, J J. Am. Chem. Soc. 2003, 125, 8270-8284. 